\title{
A UK-Wide Survey of Life-Threatening Thyroidectomy Complications
}

\author{
Z. K. Hassan-Smith, P. Gopinath, and F. Mihaimeed \\ Department of Surgery, Newham University Hospital NHS Trust, Glen Road Plaistow, London E13 8SL, UK \\ Correspondence should be addressed to F. Mihaimeed, faisal.mihaimeed@newhamhealth.nhs.uk
}

Received 13 June 2010; Revised 6 December 2010; Accepted 6 January 2011

Academic Editor: Fausto Bogazzi

Copyright (๑) 2011 Z. K. Hassan-Smith et al. This is an open access article distributed under the Creative Commons Attribution License, which permits unrestricted use, distribution, and reproduction in any medium, provided the original work is properly cited.

\begin{abstract}
Background and Aims. Complications following thyroidectomy can prolong hospital stay and cause significant morbidity particularly for patients treated for benign thyroid conditions. Our aim was to administer a UK-wide survey of thyroid surgery units on frequency and timing of the onset of life-threatening airway complications \& correlate to factors that might be associated with them. Methods. A questionnaire including the number of and timing of the onset of life-threatening airway complications, number of thyroidectomy procedures performed per year, surgeon years of experience, the use of difficult airway management protocol, post-operative patient destination, and patient deaths, was sent to $80 \mathrm{UK}$ surgical units. Results. 23/41 hospitals responded reported no postthyroidectomy airway complications. Life-threatening airways complications all occurred within the first 12 hours postoperatively, with 9 cases occurring in the recovery room and in less than 2 hours, 3 cases occurring 2-6 hours, and 3 cases occurring 6 to 12 hours after surgery. Conclusion. The results may support recent publications that advocate thyroidectomy as a less-than-24-hour surgery procedure in selected patients. Further a larger study and standardised protocol are required to establish patients' selection criteria to determine who are likely to develop serious postoperative complication and may require HDU bed.
\end{abstract}

\section{Introduction}

The morbidity and mortality of thyroidectomy are rare in occurrence, well documented, and known. Some of the immediate postoperative complications are very serious and if not dealt with promptly could be fatal. Life-threatening complications such as postoperative haemorrhage and airway obstruction fortunately occur infrequently but deserve more research to prevent mortality as result. The optimum time for close postoperative observation is undetermined, and some units have started performing some thyroid surgery as day case (less-than-24-hour hospital stay). Certain factors such as extent of resection, additional neck dissection, indication for thyroidectomy, and patient volume per surgeon significantly affect the morbidity of thyroid surgery and the length of stay [1]. Some complications such as hypocalcaemia [2-5] and recurrent laryngeal nerve palsy can be quite disturbing for patients in their permanent form and can prolong hospital stay and add to patients' morbidities [6, 7]. Research has shown that full exposure of the recurrent laryngeal nerve during the thyroidectomy can reduce the rate of morbidity associated [8]. This research has also led to the standard practice of preoperative and postoperative endoscopic assessment of vocal cords to ensure that any operative damage is detected and managed as soon as possible [1]. Furthermore, more experienced surgeons will tend to operate on the thyroid cancer patients who inherently have more complications because of distorted anatomy and difficulty in exposing the important structures especially with advanced infiltrative cancers $[9,10]$. The main aim of this study was not only to conduct a survey of thyroid units across the UK focussing particularly on the timing of development of serious postoperative complications but also to relate these to patients' volume per surgeon, years of experience, and number of thyroid surgeons per unit and to determine how the management of postoperative thyroidectomy varies and whether there is an established protocol in the units surveyed. We also looked at the patient 


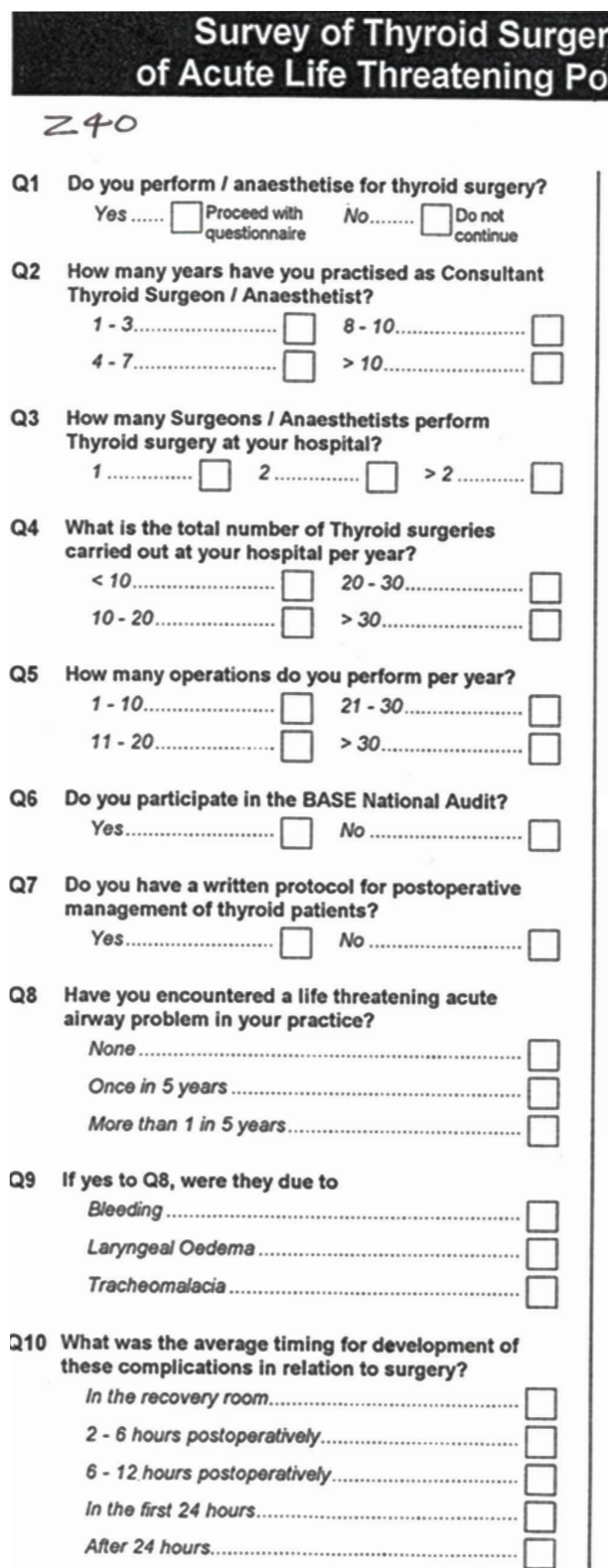
Q11 Do you have any policy for postoperative management of patient with difficult airways or large retro-sternal goitre?

Yes

No

Q12 Do you send your patients routinely to the surgical ward from recovery?

ward from recovery?

No.

Q13 Do you send your patient to HDU routinely? Yes ........... $\square$ No....

Q14 What influences your decision to send the patient to HDU?

Routine Practice . Large retro-sternal goitre Total Thyroidectomy Difficult Airways on Preoperative Assessment ... Other (please specify)

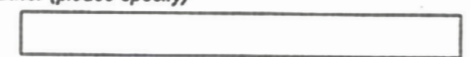

Q15 Do you keep patients ventilated following large retro -sternal goitre thyroidectomy?

Yes..

No

Q16 What influences your decision? Size of the Goiltre..

Preoperative Anaesthetic Assessment of the Airways.

Other (please specity)

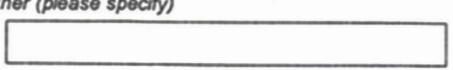

Q17 Have you encountered post thyroidectomy deaths in your unit?

Yes.

No

Q18 If Yes, what was the reason for death/s?

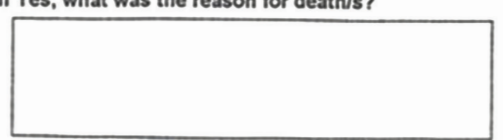

Thank you for your help. We will write back to you with the result of this analysis.

FIGURE 1: Scanned image of questionnaire used.

destination for the immediate postoperative period and details of any postoperative deaths.

\section{Methods}

A questionnaire was designed by senior author (F. Mihaimeed) (Figure 1) and was sent to 80 thyroid units in the UK. Units were identified from NHS hospital directory and by telephone calls to each of the identified hospitals and sending the questionnaire to identified named surgeons or anaesthetists who carried out thyroid surgery. The answers to each of the questions were collated and represented in graphical format.

\section{Results}

A response rate of $51 \%(41 / 80)$ was achieved. Of the 41 questionnaires received, $44 \%$ of surgeons had more than 10 years experience, $7 \%$ with $8-10$ years, $39 \%$ with $4-7$ years, and $10 \%$ with $1-3$ years (Figure 2). $44 \%$ of hospitals had more than 2 thyroid surgeons, 37\% with 2 thyroid surgeons, and 20\% with 1 thyroid surgeon. $33 / 41$ hospitals conducted more than 30 thyroid operations per year, 19 of which with more than 30 operations per surgeon per year. A proportion of respondents (23\%) perform too few operations $(<20)$ per year. 23/41 hospitals had no airway problems, with 13 hospitals experiencing 1 airway problem and 5 experiencing more than 1 (Figure 3 ). This was due to 


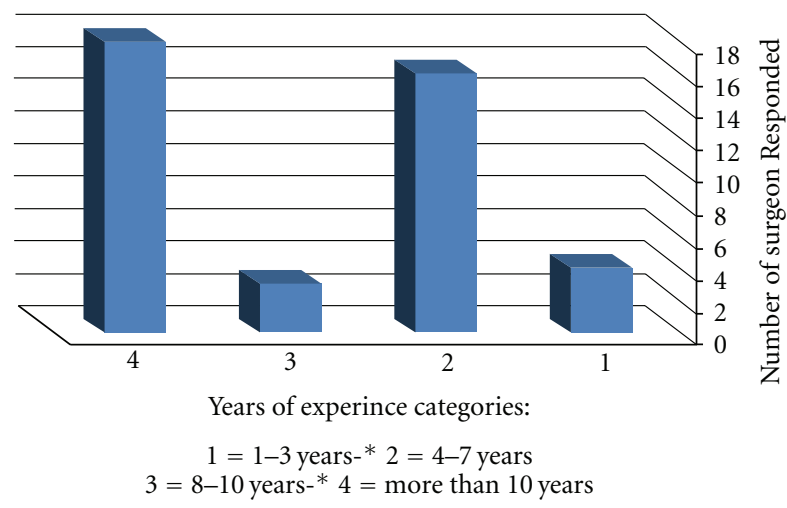

FIGURE 2: Bar chart representing the experience in thyroid surgery across the 41 units.

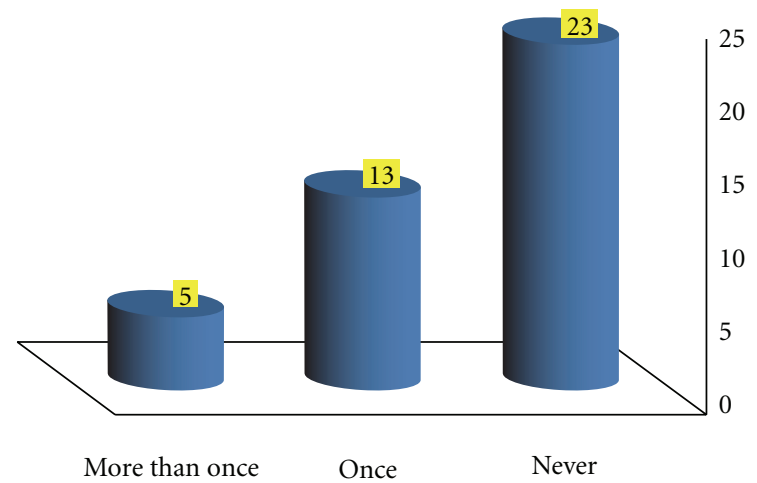

FIGURE 3: Bar chart representing the frequency of airway problems encountered.

bleeding alone (7/16), laryngeal oedema (4/16), and bleeding and oedema (4/16), and there was one case of tracheomalacia with 1 hospital that did not answer this question (Figure 4). These airway problems all occurred within the first 12 hours of postoperative period, with 9 cases occurring in the recovery room, 3 cases from 2-6 hours, and 3 cases from $6-12$ hours. $5 \%$ of units routinely ventilated patients after large retrosternal goitre thyroidectomy, and $51 \%(21 / 41)$ of units had a policy for the management of difficult airways. $98 \%$ of units routinely discharged their patients to the ward and $5 \%$ of units routinely discharged their patients to high dependency unit (HDU). There were 4 post-operative deaths: in one case the reason was not given and another suffered sudden death 2 hours after surgery and had postmortem study that confirmed cardiac event and no technical surgical complications. The other two were caused by haemorrhage from anaplastic carcinoma invasion of the carotid artery and the other by bleeding into tracheostomy after parathyroidectomy.

$5 / 41$ units had more than one airway complication in the last 5 years, and these surgeons fell into the 4-7-year, 8-10-year and more-than-10-year-experience groups, and $100 \%(4 / 4)$ of the surgeons with 1-3 years experience had no airway complications. Of the 33 units who had performed

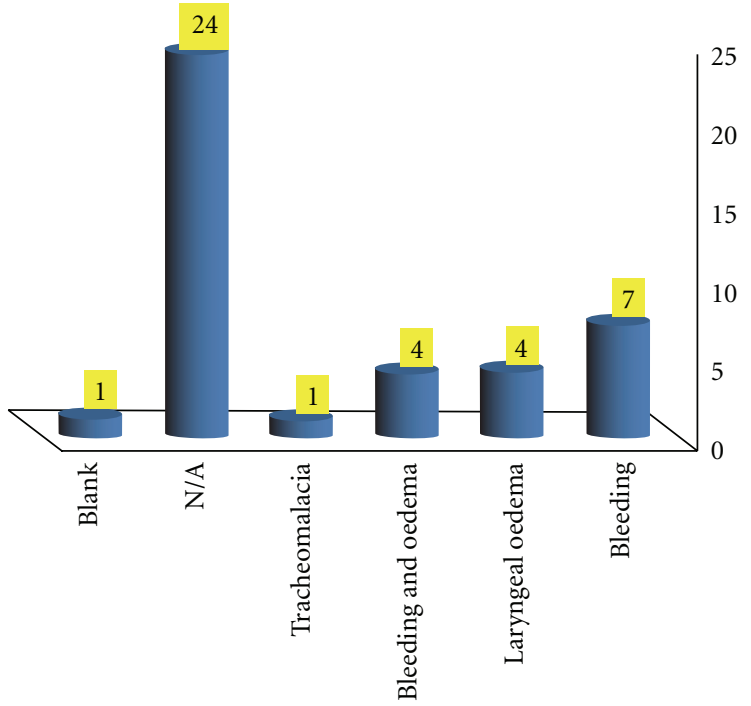

Figure 4: Bar chart representing the different causes of the airway problems.

TABLE 1: The frequency of airway complications according to the length of surgical experience.

\begin{tabular}{lccc}
\hline $\begin{array}{l}\text { Surgical } \\
\text { experience in } \\
\text { years }\end{array}$ & $\begin{array}{c}\text { More than one } \\
\text { airway } \\
\text { complication }\end{array}$ & $\begin{array}{c}\text { One airway } \\
\text { complication }\end{array}$ & $\begin{array}{c}\text { No airway } \\
\text { complications }\end{array}$ \\
\hline $1-3$ & 0 & 0 & 4 \\
$4-7$ & 2 & 4 & 10 \\
$8-10$ & 1 & 1 & 1 \\
$>10$ & 2 & 8 & 8 \\
\hline
\end{tabular}

TABLE 2: The number of thyroidectomies performed annually compared to the frequency of airway complications.

\begin{tabular}{lccc}
\hline $\begin{array}{l}\text { Number of } \\
\text { thyroidectomies } \\
\text { performed }\end{array}$ & $\begin{array}{c}\text { More than one } \\
\text { airway } \\
\text { complication }\end{array}$ & $\begin{array}{c}\text { One airway } \\
\text { complication }\end{array}$ & $\begin{array}{c}\text { No airway } \\
\text { complications }\end{array}$ \\
\hline$<10$ & 0 & 1 & 1 \\
$11-20$ & 0 & 1 & 1 \\
$21-30$ & 1 & 1 & 2 \\
$>30$ & 4 & 10 & 19 \\
\hline
\end{tabular}

more than 30 thyroidectomies, 19 had no complications (58\%), 10 had 1 airway complication (30\%), and 4 units $(12 \%)$ had more than one airway complication in the last five years. $44 \%(8 / 18)$ of the units with more than ten years experience had no airway complications, $44 \%$ $(8 / 18)$ had one airway complication, and $12 \%(2 / 18)$ had more than one airway complication (see Tables 1 and 2).

\section{Discussion}

The study demonstrates that airway complications happened within 12 hours of surgery, which would support some 
of the recent publications that advocate thyroidectomy as a less-than-24-hour surgical procedure [11]. There is a lack of uniformity in terms of using standardised protocol for management of post-operative thyroidectomy and no clear rationale or stated specific patients' selection criteria for admitting patients to HDU postoperatively. The responses suggest that the decision to admit to HDU or discharge to general ward from recovery room was subjective to anaesthetic and/or surgical opinion. Potentially, with standardised criteria, patients' selection for HDU transfer would ensure better resource utilisation. The complication rates are not higher with limited years of surgical experience, which supports previous reports. This agrees with previous literature on endocrine surgical trainees; usually these surgeons either had completed supervised period of training or would be supervised by more senior surgeon, so the likelihood of complications is reduced [12, 13]. Albeit; the small surgeons sample size in this category, and the details of patients' selection, and types of thyroid procedures performed were not included in this study, which could be a limiting factor to draw any useful conclusion in this respect.

In addition, patients' volume per surgeon needs to be taken into account along with comorbidities, age of the patient, preoperative airway assessment, extent of resection (total, near total or lobectomy, retrosternal), nature of disease (benign or malignant disease), tracheal compression, and unilateral nerve palsy before any sound conclusions on the complications' data could be concluded. Reactionary haemorrhage has been rarely previously reported after 24 hours from surgery [14]. Airway problems were mainly due to postoperative bleeding in combination with laryngeal oedema. Ensuring meticulous surgical technique and intraoperative haemostasis before wound closure and administration of prophylactic steroid injection intraoperatively in patients' with difficult dissection or complicated pathology may further decrease the incidence of postoperative serious airways complications. The use of wound drains remains to be controversial. The survey shows that there are very few post-operative deaths, which is consistent with the published reports.

\section{Conclusion}

This survey demonstrated that postthyroidectomy lifethreatening airway complications all occurred in the first 12 hours after surgery. Close patients' observation and monitoring may only be required for the first 12 hours postoperatively in the majority of postthyroidectomy patients. This data supports previous published reports and suggests that patients could be discharged home safely in less than 24 hours after thyroidectomy in selected cohort of patients. However, larger study with accurate and detailed data collection is required to address the patients' selection criteria and determine who would benefit from the short period of admission to ITU/HDU and who could be discharged safely either to general surgical ward or home within 24 hours after surgery.

\section{Acknowledgments}

The authors would like to thank Dr. Laura Ridgeway for her help with the initial preparation of this survey, Mr. Ken Johns for his help with analysing the data, and all of the units that responded and completed the questionnaire. This data was presented as abstract and oral presentation at the Annual Conference of Society for Endocrinology annual meeting 2005, London, UK British Association of Day Surgery, June 2006, Glasgow, UK and the Annual Scientific Meeting of British Association of Endocrine and Thyroid Surgeons (BAETS), September 2006, Oxford, UK. This research did not receive any specific grant from any funding agency in the public, commercial or not-for-profit sector.

\section{References}

[1] M. Steurer, C. Passler, D. M. Denk, B. Schneider, B. Niederle, and W. Bigenzahn, "Advantages of recurrent laryngeal nerve identification in thyroidectomy and parathyroidectomy and the importance of preoperative and postoperative laryngoscopic examination in more than 1000 nerves at risk," Laryngoscope, vol. 112, no. 1, pp. 124-133, 2002.

[2] A. Bergenfelz, S. Jansson, A. Kristoffersson et al., "Complications to thyroid surgery: results as reported in a database from a multicenter audit comprising 3,660 patients," Langenbeck's Archives of Surgery, vol. 393, no. 5, pp. 667-673, 2008.

[3] N. Bhattacharyya and M. P. Fried, "Assessment of the morbidity and complications of total thyroidectomy," Archives of Otolaryngology-Head and Neck Surgery, vol. 128, no. 4, pp. 389-392, 2002.

[4] F. Pattou, F. Combemale, S. Fabre et al., "Hypocalcemia following thyroid surgery: incidence and prediction of outcome," World Journal of Surgery, vol. 22, no. 7, pp. 718-724, 1998.

[5] L. Szubin, A. Kacker, R. Kakani, A. Komisar, and S. Blaugrund, "The management of post-thyroidectomy hypocalcemia," Ear, Nose and Throat Journal, vol. 75, no. 9, pp. 612-616, 1996.

[6] R. Bergamaschi, G. Becouarn, J. Ronceray, and J. P. Arnaud, "Morbidity of thyroid surgery," American Journal of Surgery, vol. 176, no. 1, pp. 71-75, 1998.

[7] A. Ríos-Zambudio, J. Rodríguez, J. Riquelme, T. Soria, M. Canteras, and P. Parrilla, "Prospective study of postoperative complications after total thyroidectomy for multinodular goiters by surgeons with experience in endocrine surgery," Annals of Surgery, vol. 240, no. 1, pp. 18-25, 2004.

[8] M. Hermann, G. Alk, R. Roka, K. Glaser, and M. Freissmuth, "Laryngeal recurrent nerve injury in surgery for benign thyroid diseases: effect of nerve dissection and impact of individual surgeon in more than 27,000 nerves at risk," Annals of Surgery, vol. 235, no. 2, pp. 261-268, 2002.

[9] H. E. Wagner and C. Seiler, "Recurrent laryngeal nerve palsy after thyroid gland surgery," British Journal of Surgery, vol. 81, no. 2, pp. 226-228, 1994.

[10] O. H. Clark, "Total thyroidectomy. The treatment of choice for patients with differentiated thyroid cancer," Annals of Surgery, vol. 196, no. 3, pp. 361-370, 1982.

[11] A. Shaha, B. M. Jaffe, I. B. Rosen, and J. N. Atti, "Complications of thyroid surgery performed by residents," Surgery, vol. 104, no. 6, pp. 1109-1114, 1988.

[12] J. K. Harness, L. Fung, N. W. Thompson, R. E. Burney, and M. K. McLeod, "Total thyroidectomy: complications and 
technique," World Journal of Surgery, vol. 10, no. 5, pp. 781786, 1986.

[13] A. Mishra, G. Agarwal, A. Agarwal, and S. K. Mishra, "Safety and efficacy of total thyroidectomy in hands of endocrine surgery trainees," American Journal of Surgery, vol. 178, no. 5, pp. 377-380, 1999.

[14] R. Mirnezami, A. Sahai, A. Symes, and T. Jeddy, "Daycase and short-stay surgery: the future for thyroidectomy?" International Journal of Clinical Practice, vol. 61, no. 7, pp. 1216-1222, 2007. 


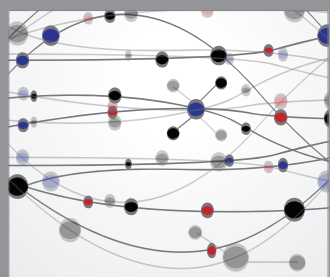

The Scientific World Journal
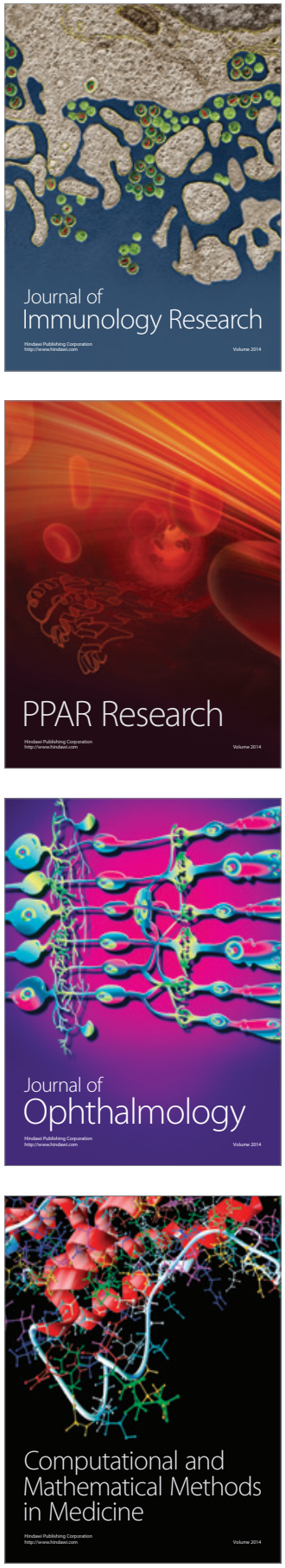

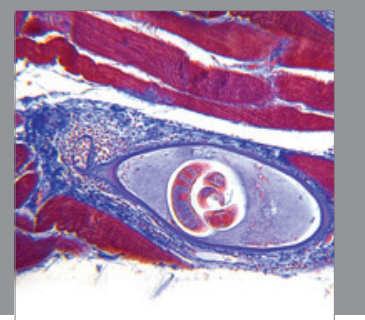

Gastroenterology

Research and Practice
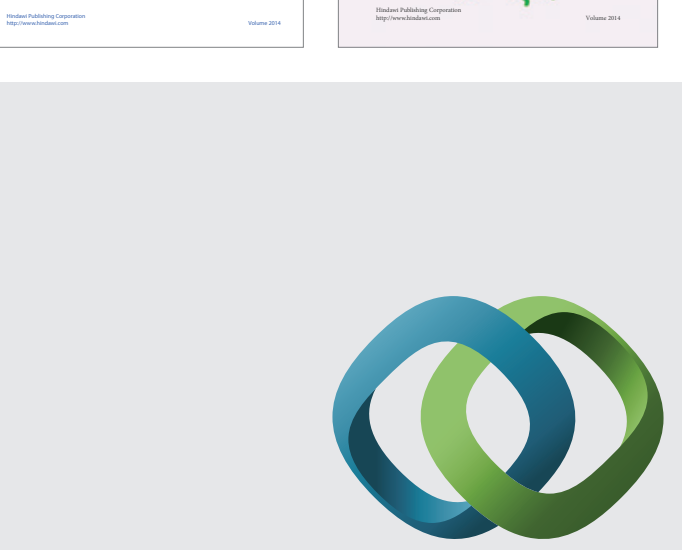

\section{Hindawi}

Submit your manuscripts at

http://www.hindawi.com
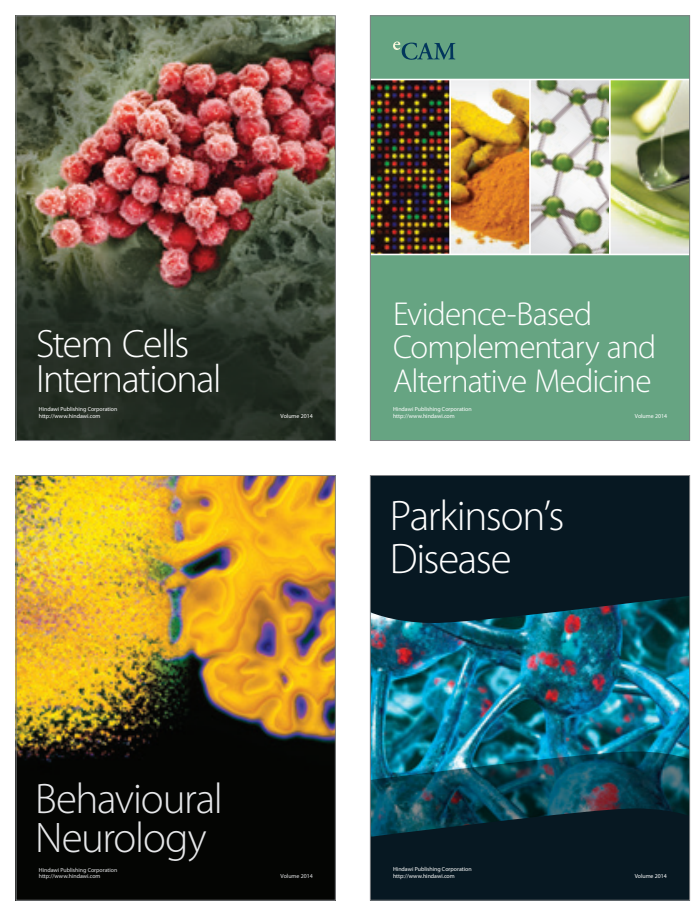

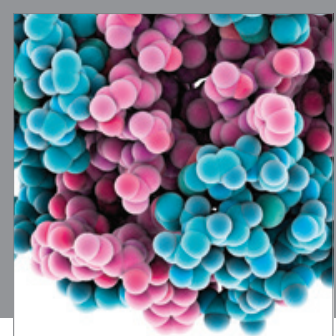

Journal of
Diabetes Research

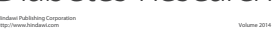

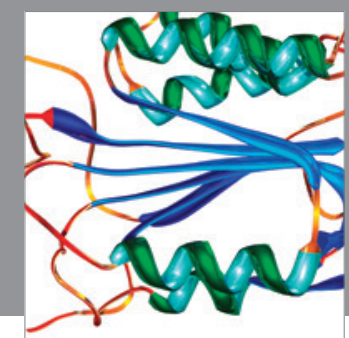

Disease Markers
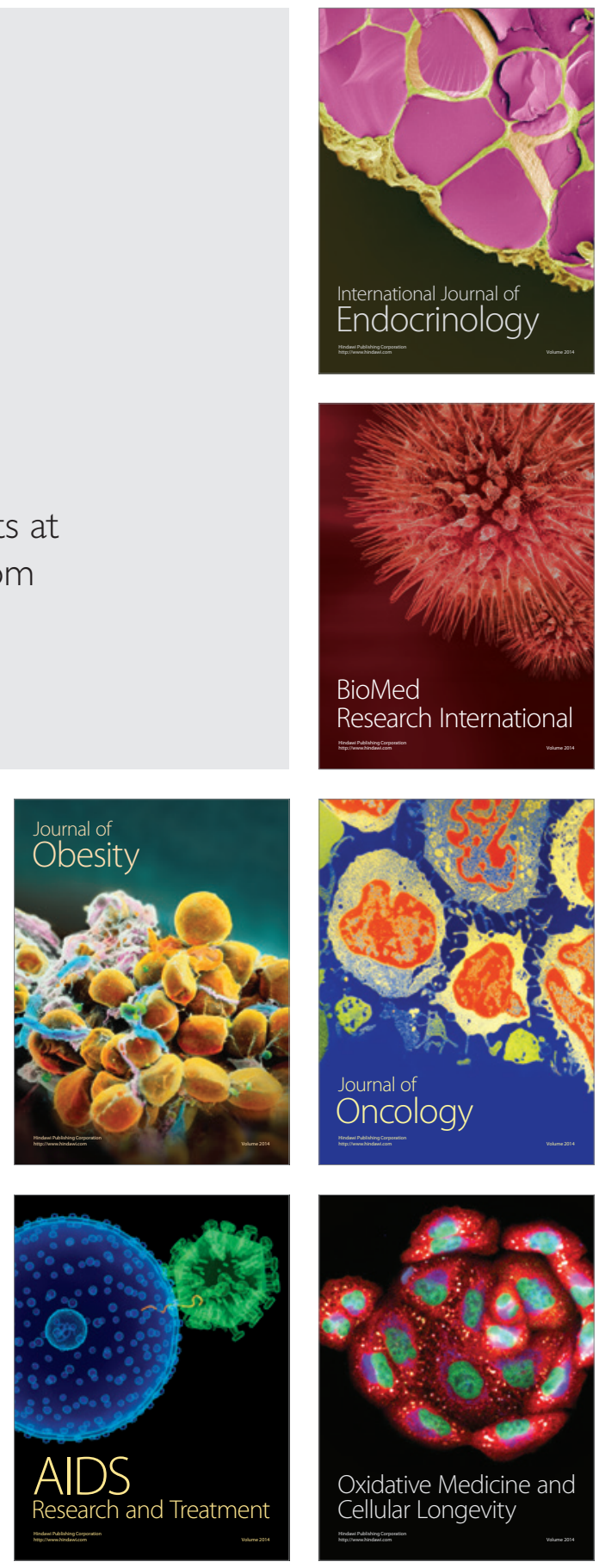